\title{
Spacetime Defects: Torsion Loops
}

\author{
Patricio S. Letelier由 \\ Departamento de Matemática Aplicada-IMECC \\ Universidade Estadual de Campinas \\ 13081-970 Campinas. S.P., Brazil
}

April 19, 1995

\begin{abstract}
Spacetimes with everywhere vanishing curvature tensor, but with torsion different from zero only on world sheets that represent closed loops in ordinary space are presented, also defects along open curves with end points at infinity are studied. The case of defects along timelike loops is also considered and the geodesics in these spaces are briefly discussed.
\end{abstract}

PACS numbers: 98.80.Cq, 04.20 Jb, 04.40.+c, 11.17.+y

\footnotetext{
${ }^{1}$ e-mail: letelier@ime.unicamp.br
} 
Conical singularities or spacetime defects are characterized by RiemannChristoffel curvature tensor, or Cartan torsion, or both different from zero only on the subspace (event, world line, world sheet, or world tube) that describes the evolution of the defect (texture, monopole, string, or membrane). In other words we have that the curvature, the torsion, or both, are proportional to distributions with support on the defect. Spacetimes with conical singularities of different types has been studied recently in a variety of contexts, e.g. spinning strings with cosmic dislocations [1] [2], pure spacetime dislocations [3] and [4], also in low dimensional gravity [5]. For the discussion of a great variety of defects see Ref. [6].

The already known cases of line defects, in usual space, are infinite strait line defects with or without torsion. The case of constant torsion was studied in [罒]. Lines of dislocations that grow linearly with time and also spin that grows linearly along the defect were studied in [3] and [4. It is worth to mention that following the same methodology of [2] one can also super impose to this defect the defect angle that represents a disclination (cosmic string). The purpose of this letter is to study spacetimes with torsion line defects of arbitrary shape.

Let us consider the metric

$$
d s^{2}=\left(\omega^{0}\right)^{2}-\left(\omega^{1}\right)^{2}-\left(\omega^{2}\right)^{2}-\left(\omega^{3}\right)^{2},
$$


with

$$
\begin{aligned}
\omega^{0} & =d t+B_{x} d x+B_{y} d y+B_{z} d z, \\
& =d t+\mathbf{B} \cdot d \mathbf{x} \\
\omega^{1} & =d x, \omega^{2}=d y, \omega^{3}=d z
\end{aligned}
$$

where $\mathbf{B}=\left(B_{x}, B_{y}, B_{z}\right)$ are arbitrary functions of the coordinates $\mathbf{x}=$ $(x, y, z)$ only. Let us assume that the spacetime has a torsion of the form,

$$
\begin{aligned}
& T^{0}=J_{z} \omega^{1} \wedge \omega^{2}+J_{x} \omega^{2} \wedge \omega^{3}+J_{y} \omega^{3} \wedge \omega^{1}, \\
& T^{1}=T^{2}=T^{3}=0,
\end{aligned}
$$

where $\mathbf{J}=\left(J_{x}, J_{y}, J_{z}\right)$ are functions of $\mathbf{x}=(x, y, z)$ only. From the first Cartan structure equation,

$$
\underline{\mathrm{T}}^{a}=d \omega^{a}+\omega^{a}{ }_{b} \wedge \omega^{b},
$$

we find the connection one-forms,

$$
\begin{aligned}
2 \omega^{01} & =\left(\partial_{x} B_{y}-\partial_{y} B_{x}-J_{z}\right) \omega^{2}+\left(\partial_{z} B_{x}-\partial_{x} B_{z}-J_{y}\right) \omega^{3}, \\
2 \omega^{02} & =-\left(\partial_{x} B_{y}-\partial_{y} B_{x}-J_{z}\right) \omega^{1}+\left(\partial_{y} B_{z}-\partial_{z} B_{y}-J_{x}\right) \omega^{3}, \\
2 \omega^{12} & =\left(\partial_{x} B_{y}-\partial_{y} B_{x}-J_{z}\right) \omega^{0}, \\
2 \omega^{23} & =\left(\partial_{y} B_{z}-\partial_{z} B_{y}-J_{x}\right) \omega^{0}, \\
2 \omega^{31} & =\left(\partial_{z} B_{x}-\partial_{x} B_{z}-J_{y}\right) \omega^{0} .
\end{aligned}
$$


Now, we shall postulate that the "Cartesian" vectors $\mathbf{B}$ and $\mathbf{J}$ are related by the Cartesian equation,

$$
\nabla \times \mathbf{B}=\mathbf{J} .
$$

Then, we have that the connection one-forms are zero, $\omega^{a b}=0$, and as a consequence of the second Cartan structure equations,

$$
R_{b}^{a}=d \omega_{b}^{a}+\omega^{a}{ }_{c} \wedge \omega_{b}^{c},
$$

the curvature two-forms are also zero, $R^{a b}=0$. Thus we have a spacetime with zero curvature and non vanishing torsion, i.e., a Weitzenböck space [8] We can choose the torsion (33) as a distribution with support along a curve $C$ with parametric equation $\mathbf{x}^{\prime}=\mathbf{x}^{\prime}(\lambda)$ taking the function $\mathbf{J}$ as

$$
\mathbf{J}(\mathbf{x})=I \int_{C} \delta^{(3)}\left(\mathbf{x}-\mathbf{x}^{\prime}(\lambda)\right) \frac{d \mathbf{x}^{\prime}(\lambda)}{d \lambda} d \lambda,
$$

where $I$ is an arbitrary constant. The components of $\mathbf{J}$ can also be written in the more appealing form [7],

$$
J_{k}(\mathbf{x})=I \delta_{k}^{(2)}\left(\mathbf{x}_{\perp}, C\right) .
$$

The function (8) isline $C$ that generalizes to an arbitrary curve the formula valid for a strait line of torsion placed along the $z$-axis

$$
J_{z}=I \int \delta(x) \delta(y) \delta\left(z-z^{\prime}\right) d z^{\prime}=I \delta(x) \delta(y) .
$$


The integrability condition for equation (6) is $\nabla \cdot \mathbf{J}=0$; from (8) we get,

$$
\nabla \cdot \mathbf{J}=\delta^{(3)}\left(\mathbf{x}-\mathbf{x}_{i}^{\prime}\right)-\delta^{(3)}\left(\mathbf{x}-\mathbf{x}_{f}^{\prime}\right)
$$

Thus to satisfy this condition we need either that the initial and final points of the curve coincide, $\mathbf{x}_{i}^{\prime}=\mathbf{x}_{f}^{\prime}$, i.e., a closed curve, or that the two end points of $C$ be at infinity. In both cases we find that the solution of (6) is given by the well known formula,

$$
\mathbf{B}=\frac{I}{4 \pi} \int_{C} \frac{d \mathbf{x}^{\prime} \times\left(\mathbf{x}-\mathbf{x}^{\prime}\right)}{\mid\left(\mathbf{x}-\left.\mathbf{x}^{\prime}\right|^{3}\right.} .
$$

The metric (11) built with the forms (2) with the functions $\mathbf{B}$ given above have zero curvature tensor and non vanishing torsion along the curve $C$ of the usual space. In the four dimensional space we have a cylindrical surface with generators parallel to the $t$-axis that pierce the hyperplane $t=$ constant in $C$.

Another spacetime with the same characteristics is (1) with the oneforms

$$
\begin{aligned}
& \omega^{0}=d t, \omega^{1}=d x, \omega^{2}=d y \\
& \omega^{3}=d z+E_{x} d x+E_{y} d y+E_{t} d t,
\end{aligned}
$$

where $\mathbf{E}=\left(E_{x}, E_{y}, E_{t}\right)$ are arbitrary functions of the coordinates $(x, y, t)$, and torsion,

$$
T^{0}=T^{1}=T^{2}=0,
$$




$$
T^{3}=K_{t} \omega^{1} \wedge \omega^{2}+K_{x} \omega^{2} \wedge \omega^{0}+K_{y} \omega^{0} \wedge \omega^{1}
$$

where the functions $\mathbf{K}=\left(K_{x}, K_{y}, K_{t}\right)$ are arbitrary functions connection one-forms gives us,

$$
\tilde{\nabla} \times \mathbf{E}=\mathbf{K}, \quad\left(\tilde{\nabla}=\left(\partial_{x}, \partial_{y}, \partial_{t}\right)\right)
$$

Therefore, solutions of this last equation for a torsion built with a function $\mathbf{K}$ similar to (8) but with the coordinate $z$ changed by $t$ are spacetimes with everywhere vanishing curvature tensor, and torsion given by a distribution with support on a curve $\tilde{C}$ in the three space $(x, y, t)$. In the four dimensional spacetime this curve are lifted to a cylindrical surface with generators parallel to the $z$-axis.

Now we want to consider test particles moving in these spacetimes with torsion defects. We have two different equations of motion: the autoparallels and the geodesics. Since, in both classes of spacetimes we have zero Riemann-Cartan connection, autoparallels are strait lines. For the first type of defects we have $\nabla \times \mathbf{B}=0$ outside the defect. Then the geodesic equation, in this case, reduces to

$$
\dot{t}+\mathbf{B} \cdot \dot{\mathbf{x}}=W, \ddot{\mathbf{x}}=0,
$$

where the dots denote differentiation with respect the proper time $s ; W$ is an integration constant. Hence, a particle following a geodesic travels along 
a strait line, in general, with variable speed. For the second case, outside the defect we also have $\tilde{\nabla} \times \mathbf{E}=0$ and the geodesic equation gives us,

$$
\begin{aligned}
& \ddot{t}=\ddot{x}=\ddot{y}=0, \\
& \dot{z}=\tilde{W}-E_{x} \dot{x}-E_{y} \dot{y}-E_{t} \dot{t},
\end{aligned}
$$

where $\tilde{W}$ denotes another integration constant. In this case the geodesics are plane curves, the $z$-axis is also contained on the plane defined by the curve.

In summary, we havby everywhere zero Riemann-Christoffel curvature tensor and non vanishing torsion. The torsion can be concentrated on a closed line or on an open line with end points at infinity. Ideas for a field theoretical approach to this defects were advanced in [2], we hope to comeback to this point in another occasion. 


\section{References}

[1] D.V. Gal'tsov and P.S. Letelier, Phys. Rev. D 47, 4273 (1993).

[2] P.S. Letelier, Class. Quantum Grav. 12, 471-478 (1995).

[3] K.P. Tod, Class. Quantum Grav. 11, 1331 (1994).

[4] D.G.B. Edelen, Int. J. Theor. Phys. 35, 1315 (1994).

[5] C. Kohler, Class. Quantum Grav. 12, L11 (1995).

[6] P.S. Letelier and A. Wang, Spacetime Defects, J. Math. Phys. in press; P.S. Letelier, Spacetime Defects: Open and Closed Shells, ibid.

[7] See for instance, H. Kleinert, Gauge Fields in Condensed Matter, (World Scientific, Singapura 1989).

[8] See for instance, J.A. Schouten, Ricci-Calculus (Springer, Berlin 1954). 